\section{Ten-Year Plateau Phase in Human Immunodeficiency Virus Induced Motor Neuron Disease upon Antiretroviral Therapy: A First Case from Eastern Africa Marjolein E. de Bruin, MD ${ }^{1,2}$, Emmanuel V. Assey, MD', Asha Osman, $\mathrm{MD}^{1}$, Kajiru Kilonzo, $\mathrm{MD}^{1}$, William P. Howlett, MD', and Marieke C.J. Dekker, MD ${ }^{1}$ 'Department of Medicine, Kilimanjaro Christian Medical Centre, P.O. Box 3010, Moshi, United Republic of Tanzania. ${ }^{2}$ Department of Neurology, Haaglanden Medisch Centrum, PO Box 4322501 CK The Hague, Netherlands.}

\section{ABSTRACT}

We report an individual with rapidly progressive motor neuron disease (MND), phenotypically compatible with amyotrophic lateral sclerosis (ALS). The patient described in this case report proved positive for human immunodeficiency virus (HIV) and was initiated on antiretroviral therapy (ART). Following ART he clinically stabilised over 10 years and deteriorated again due to noncompliance or ART resistance. HIV infection can give rise to an MND mimic, HIV-ALS. The improvement in response to ART supports the notion that HIV-ALS is a treatable entity also in Africa. This is the first case report of a patient with HIV-ALS and long term follow up in Sub-Saharan Africa. The report raises the suggestion that an additional (retro)virus can play a role in the aetiology of ALS.

\section{Introduction}

Patterns of neurological morbidity and mortality in low-income countries, like the Sub-Saharan African (SSA) country of Tanzania, differ in comparison to high income countries, because of a greater burden of communicable diseases., In SSA, the epidemiology and phenotypes of non-communicable neurological conditions like neuromuscular diseases are likely comparable to other populations across the world, although underdiagnosed and -reported. ${ }^{1-3}$ While motor neuron disease (MND) has been reported from SSA since the nineteen seventies, there have been few recent publications. ${ }^{3-7}$

Human immunodeficiency virus (HIV) infection is still one of the leading causes of morbidity and mortality in the world and its neurological manifestations are mani- fold. ${ }^{89}$ Since early on in the HIV epidemic, multiple cases of HIV-ALS have been described. ${ }^{10-16}$ HIV-ALS can cause a clinical presentation compatible with sporadic ALS (sALS). [15] HIV-ALS tends to occur at younger age, with a more severe and progressive phenotype.,10-15 The occurrence of HIV-ALS is not related to the duration or stage of the HIV infection. ${ }^{14}$

SSA accounts for over 70\% of the global HIV epidemic. There has been no literature on HIV-ALS from SSA apart from a recent study from South Africa, a country with higher socioeconomic level and a multi-ethnic patient population.? An association of HIV and MND was suggested in a large cohort of MND patients from Northern Tanzania.. ${ }^{4}$ One patient from the same series with HIV-ALS is described in this report. He was treated with ART and clinically stabilised over 10 years.

In patients with HIV-ALS a response to ART has been described in previous case reports. ${ }^{710-15}$ The diagnostic consideration of HIV-ALS is important since treatment with ART can affect the course of the disease.

\section{Case report}

In June 2007, a 41 year old male was admitted to Kilimanjaro Christian Medical Centre (KCMC), a tertiary referral centre in Northern Tanzania. He had a nine month history of a nasal speech, progressive difficulty chewing and swallowing for seven months and difficulty walking for six months. Around the same time he had developed a seizure disorder characterized by tonic-clonic seizures which were suppressed on phenytoin. His past medical history was remarkable for the diagnosis of HIV in May 2005. In October 2006 his CD 4 count was 250 cells/cmm and he was started on ART (stavudine (d4T), lamivudine (3TC) and nevirapine (NVP)).

In June 2007, neurological examination showed reduced gag reflex, brisk jaw jerk, positive snout and palmomental reflexes. The tongue was atrophic with fasciculations, decreased range of movement and there was pseudobulbar speech. The upper limbs showed symmetrical claw like hand deformity with marked wasting and fasciculations of the forearm and hand muscles. Power was symmetrically reduced to Medical Research Council grades $3-4 / 5$ with a proximodistal gradient and more in extensor than flexor-musculature. The lower limbs were affected with marked wasting and slightly reduced power distally. All tendon reflexes were pathologically brisk with bilateral extensor plantar reflexes and Hoffman's sign. Gait was spastic 
with the need for assistance to mobilize. Sensory examination was normal. (Figure 1)

Full blood count, erythrocyte sedimentation rate (ESR) and renal and liver function tests were normal with a negative VDRL test and a CD4 count of 697 cells/cmm. Chest he could walk and function independently. His swallowing had also improved though his speech remained unchanged. From two years before the admission in 2017 he required assistance when walking. Neurological examination in 2017 was largely comparable to 2007 apart from slightly more
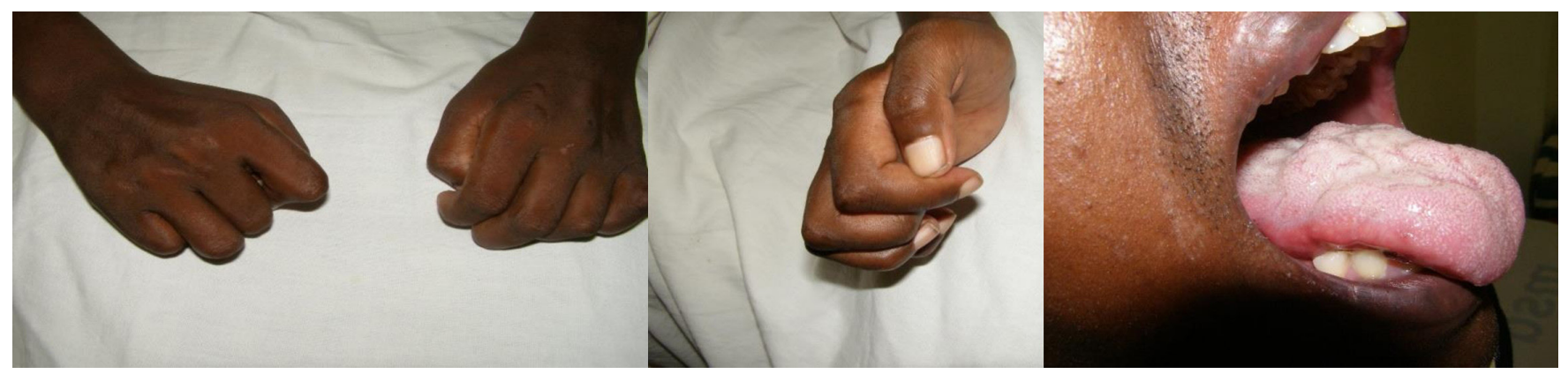

Figure 1: photos of the hands from 2008 show wasting of hand muscles and clawing. Photo of the tongue from 2008 showing atrophy.

X-ray and CT brain were normal. Cerebrospinal fluid (CSF) had normal biochemistry and cell count, and an undetectable HIV ribonucleic acid (RNA) viral load. No other cause was found for his new onset seizure disorder. There was no EMG or MRI facility in Northern Tanzania in 2007/8.

A diagnosis of HIV-ALS was made and his ART regime was changed to abacavir (ABC), didanosine (ddl) and lopinavir/ritonavir (LPV/r) for better central nervous system (CNS) penetration. He developed distal sensory neuropathy and changed ART again in December 2007 (Zidovudine (AZT), 3TC and LPV/r). He was observed with threemonthly visits, with stable symptoms until January 2008, when he was lost to follow-up.

November 2017, the patient was readmitted to KCMC with symptoms of congestive cardiac failure. He was reviewed by the same neurologist (WPH) as on his first admission. After 2008 his symptoms had improved such that pronounced upper motor neuron signs (spasticity) in his upper limbs. (Figure 2)

Over the previous 10-year period the patient said to be compliant with his ART. During the admission in 2017 plasma HIV RNA copies were 7919/ml suggesting lack of ART compliance or the emergence of ART resistance. Electromyography supported the presence of lower motor neuron degeneration in the cranial, cervical, thoracic and lumbosacral segments, based on the Awaji criteria.

Based on the history and physical examination the clinical diagnosis was 'definite' ALS. It had stabilised on ART for a decade but deteriorated with signs of therapy failure as a possible explanation. (Figure 3)

\section{Discussion}

This is the first case report of a patient in SSA, outside South Africa, with HIV-ALS. The first tentative report of

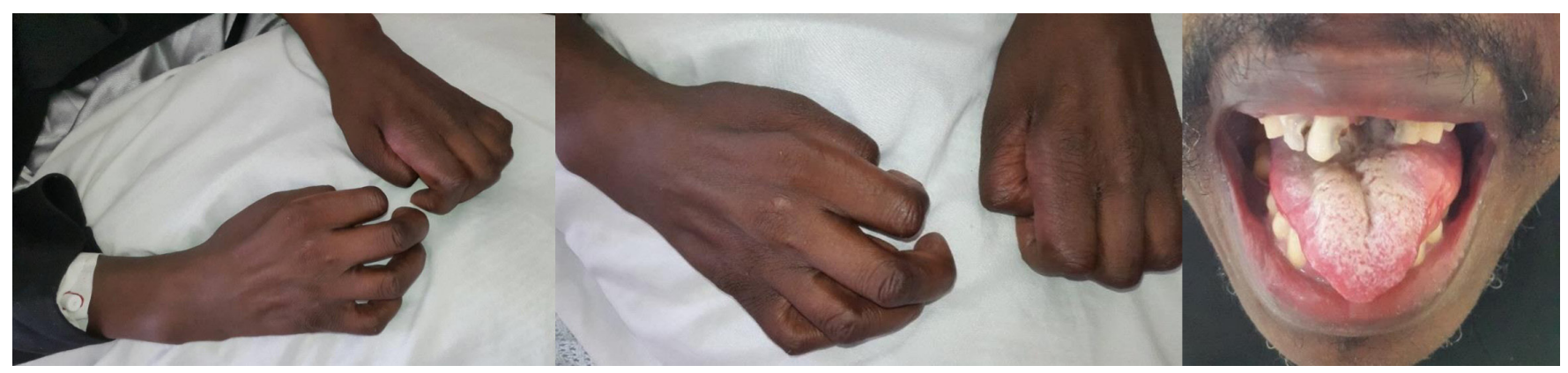

Figure 2: photos of the hands from 2017 show wasting of hand muscles and clawing. Photo of the tongue from 2017 showing atrophy. 


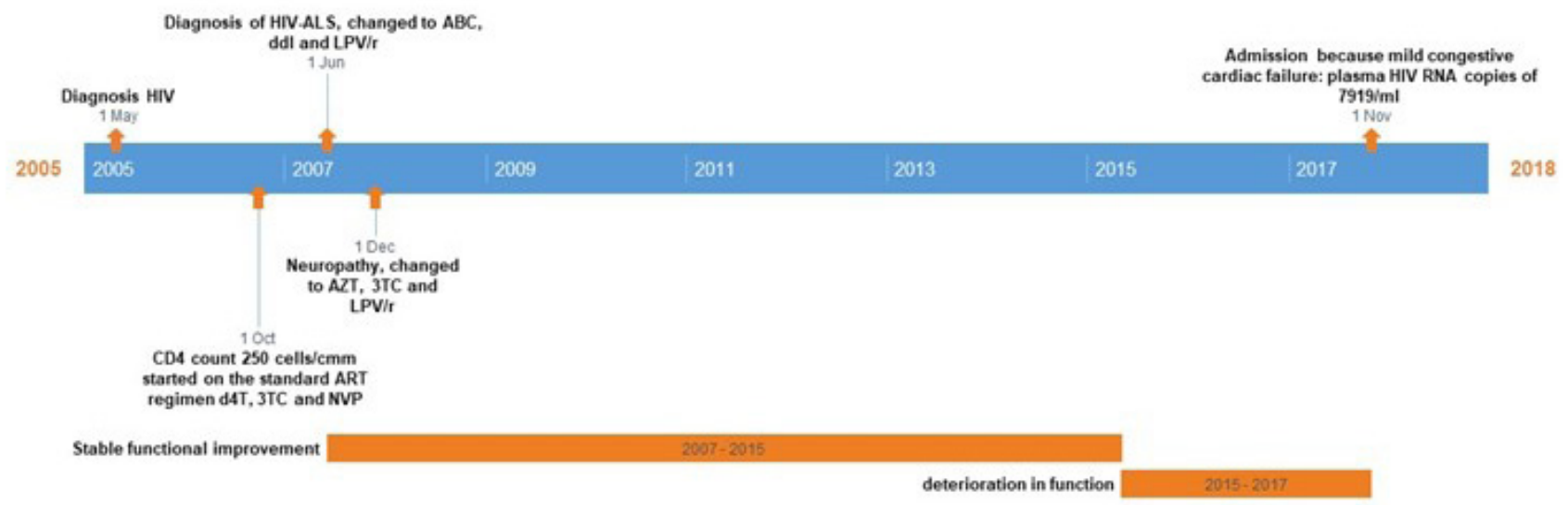

Figure 3: timeline. 3TC: lamivudine. ABC: abacavir. ALS: amyotrophic lateral sclerosis. ART: antiretroviral therapy. CD4: cluster of differentiation 4. d4T: stavudine. ddI: didanosine. HIV: Human immunodeficiency virus. LPV/r: lopinavir/ritonavir

an association between HIV and MND was of a HIV infected patient, who developed classic ALS in $1985 .{ }^{16}$ From 1985 to 2018 there have been over 30 cases described in case reports and series of HIV-associated MND. ${ }^{10-16}$ A recent retrospective study from South Africa describes 35 patients with HIV-associated motor neuron syndrome compared to 101 HIV-uninfected MND patients. ${ }^{7}$

The estimated HIV prevalence rate in adults in Tanzania is $4.6 \% .^{17}$ The global incidence of ALS is 1-2 per 100,000 persons each year with a prevalence of 4-6 cases per 100,000. ${ }^{18}$ The reported MND prevalence rates among Black African HIV-infected and HIV-uninfected patients are respectively 2.4 and 0.44 cases per 100,000.,19 HIV was suggested to be a causal confounder.,19

Clinically, HIV-ALS is identical to sALS in HIV-negative patients but occurs younger, is more severe and progressive. It can be arrested or (partially) reversed by initiation or optimisation of ART.,10-15

In our patient neurological symptoms developed on the standard ART regimen despite virological suppression. He had started ART when the CD4 count went below 250 cells/cmm, as was usual at the time. June 2007 he changed to ART with good CNS penetration, on which there was no progression of symptoms during 8 months. He was lost to follow-up for almost 10 years during which he describes a long period (7 years) of gradual improvement. From 2015 there was deterioration in function and on re-evaluation in late 2017 he was found to have a high viral load in plasma. This suggests treatment failure due to noncompliance or ART resistance. Of clinical interest was the nearly identi- cal pattern of neurological signs over the 10-year period of observation.

Partial to full clinical improvement after starting ART has also been reported in a number of studies on MND in HIV., ${ }^{710-15}$ In a South African study, 17 of 35 patients with HIV-associated motor neuron syndrome survived longer than 10 years after starting ART. Early ART initiation or optimisation ( $\leq 6$ months) and use of ART with CNS penetration improve outcome. ${ }^{7,10,14}$

The described patient developed a seizure disorder (generalised) around the same time as he started his MND symptoms, no cause was found. While the occurrence of seizures is increased significantly in patients with HIV, they are usually secondary to opportunistic processes or vascular in origin, neither of which were present in this case. Possibly there is a relation and to reclassify the diagnosis to ALS-plus is very reasonable.

A (retro)viral aetiology in sALS has been hypothesized. ${ }^{19-22}$ Motor neuron cells are vulnerable to certain viruses, illustrated by poliomyelitis virus which targets anterior horn cells. HIV can occur in the microglial cells of the CNS but is not identified to invade motor neurons. Therefore the pathophysiology of HIV-ALS might be explained by a secondary inflammatory response or an unknown opportunistic virus infecting the motor neurons. ${ }^{21}$ In some patients with sALS an increased HERV-K expression has been found in cortical neurons and anterior horn cells suggesting that this endogenous retrovirus might play a role in the aetiology ${ }^{10,11,20,22}$ The serum of this patient could unfortunately not be tested for HERV-K. 
Our first report from Tanzania supports the notion that HIV-ALS can be a treatable entity also in Africa. ${ }^{719}$

\section{Teaching points}

- HIV infection can give rise to motor neuron disease including HIV-ALS.

- HIV should routinely be tested in patients with ALS.

- Antiretroviral therapy can potentially reverse and treat HIV-ALS making it a treatable entity, also in Africa.

- Retroviruses might play a role in the aetiology of motor neuron disease.

\section{Acknowledgements}

We would like to thank the family for their participation and informed consent for the photographs, EMG and publication of this case report. We would also like to thank Kilimanjaro Christian Medical Centre for allowing us to work closely with patient and family.

\section{Corresponding Author:}

Marjolein E. de Bruin, Department of Neurology, Haaglanden Medisch Centrum,

PO Box 4322501 CK The Hague, Netherlands m.de.bruin2@haaglandenmc.nl'

\section{References}

${ }^{1}$ Disease GBD, Injury I, Prevalence C. Global, regional, and national incidence, prevalence, and years lived with disability for 310 diseases and injuries, 1990-2015: a systematic analysis for the Global Burden of Disease Study 2015. Lancet 2016; 388(10053): 1545-602.

${ }^{2}$ Dekker MCJ, Urasa SJ, Howlett WP. Neurological letter from Kilimanjaro. Pract Neurol 2017; 17(5): 412-6.

${ }^{3}$ Quansah E, Karikari TK. Motor Neuron Diseases in Sub-Saharan Africa: The Need for More Population-Based Studies. Biomed Res Int 2015; 2015: 298409.

${ }^{4}$ Dekker MCJ, Urasa SJ, Aerts MB, Howlett WP. Motor neuron disease in sub-Saharan Africa: case series from a Tanzanian referral hospital. J Neurol Neurosurg Psychiatry 2018; 89(12): 1349-50.

${ }^{5}$ Spillane JD. Tropical neurology. Proc R Soc Med 1969; 62(4): 403-10.

${ }^{6}$ Osuntokun BO. The pattern of neurological illness in tropical Africa. Experience at Ibadan, Nigeria. J Neurol Sci 1971; 12(4): 417-42.
${ }^{7}$ Moodley K, Bill PLA, Bhigjee AI, Patel VB. A comparative study of motor neuron disease in HIV-infected and HIV-uninfected patients. J Neurol Sci 2019; 397: 96-102.

${ }^{8}$ Howlett WP. Neurological disorders in HIV in Africa: a review. Afr Health Sci 2019; 19(2): 1953-77.

${ }^{9}$ Ghosn J, Taiwo B, Seedat S, Autran B, Katlama C. Hiv. Lancet 2018; 392(10148): 685-97.

${ }^{10}$ Bowen LN, Tyagi R, Li W, Alfahad T, Smith B, Wright $\mathrm{M}$, et al. HIV-associated motor neuron disease: HERV-K activation and response to antiretroviral therapy. Neurology 2016; 87(17): 1756-62.

${ }^{11}$ Alfahad T, Nath A. Retroviruses and amyotrophic lateral sclerosis. Antiviral Res 2013; 99(2): 180-7.

${ }^{12}$ Moulignier A, Moulonguet A, Pialoux G, Rozenbaum W. Reversible ALS-like disorder in HIV infection. Neurology 2001; 57(6): 995-1001.

${ }^{13}$ MacGowan DJ, Scelsa SN, Waldron M. An ALS-like syndrome with new HIV infection and complete response to antiretroviral therapy. Neurology 2001; 57(6): 1094-7.

${ }^{14}$ Verma A, Berger JR. ALS syndrome in patients with HIV-1 infection. J Neurol Sci 2006; 240(1-2): 59-64.

${ }^{15}$ Lorenzoni PJ, Ducci RD, Dalledone GO, Kay CSK, de Almeida SM, Werneck LC, et al. Motor neuron disease in patients with HIV infection: Report of two cases and brief review of the literature. Clin Neurol Neurosurg 2018; 171: $139-42$.

${ }^{16}$ Hoffman PM, Festoff BW, Giron LT, Jr., Hollenbeck LC, Garruto RM, Ruscetti FW. Isolation of LAV/HTLV-III from a patient with amyotrophic lateral sclerosis. $N$ Engl $J$ Med 1985; 313(5): 324-5.

${ }^{17} \mathrm{HIV}$ and AIDS in Tanzania. (www.unaids.org2019).

${ }^{18}$ Orsini M, Oliveira AB, Nascimento OJ, Reis CH, Leite MA, de Souza JA, et al. Amyotrophic Lateral Sclerosis: New Perpectives and Update. Neurol Int 2015; 7(2): 5885.

${ }^{19}$ Mochan A. HIV related motor neuron disease/syndrome: The - potentially treatable - retroviral link in ALS? $J$ Neurol Sci 2019; 397: 75-6.

${ }^{20}$ Douville R, Liu J, Rothstein J, Nath A. Identification of active loci of a human endogenous retrovirus in neurons of patients with amyotrophic lateral sclerosis. Ann Neurol 2011; 69(1): 141-51.

${ }^{21}$ Jubelt B, Berger JR. Does viral disease underlie ALS? Lessons from the AIDS pandemic. Neurology 2001; 57(6): 945-6.

${ }^{22}$ Li W, Lee MH, Henderson L, Tyagi R, Bachani M, Steiner J, et al. Human endogenous retrovirus-K contributes to motor neuron disease. Sci Transl Med 2015; 7(307): 307ral53. 